\title{
Fatty acid consumption and incident type 2 diabetes: an 18-year follow-up in the female E3N (Etude Epidémiologique auprès des femmes de la Mutuelle Générale de l'Education Nationale) prospective cohort study
}

\author{
Courtney Dow ${ }^{1,2}$, Marie Mangin ${ }^{1,2}$, Beverley Balkau ${ }^{1,2,3}$, Aurélie Affret ${ }^{1,2}$, \\ Marie-Christine Boutron-Ruault ${ }^{1,2}$, Françoise Clavel-Chapelon ${ }^{1,2}$, Fabrice Bonnet ${ }^{1,2,4}$ and \\ Guy Fagherazzi ${ }^{1,2 *}$ \\ ${ }^{1}$ Gustave Roussy, F-94805, Villejuif, France \\ ${ }^{2}$ Université Paris-Saclay, Université Paris-Sud, UVSQ, Inserm U1018, CESP, Health Across Generations Team, F-94805, \\ Villejuif, France \\ ${ }^{3}$ Université Versailles, F-78000, Saint Quentin, France \\ ${ }^{4}$ CHU de Rennes, Department of Endocrinology, Diabetes and Nutrition, Hôpital Sud, 16 Boulevard de Bulgarie, F-35033, \\ Rennes, France \\ (Submitted 1 June 2016 - Final revision received 28 September 2016 - Accepted 10 October 2016 - First published online 15 November 2016)
}

\section{Abstract}

We evaluated the association between dietary estimates of fatty acid (FA) consumption and type 2 diabetes (T2D) risk in the French E3N (Etude Epidémiologique auprès des femmes de la Mutuelle Générale de l'Education Nationale) cohort. In total, 71334 women without diabetes at baseline were followed up from 1993 to 2011. Diabetes was identified using questionnaires and drug-reimbursement claims, and incident cases were validated. FA consumption in 1993 was estimated from a validated dietary questionnaire. Cox regression estimated hazard ratios (HR) and $95 \%$ CI of diabetes risk, comparing the upper tertile group with the lowest. High $n-3$ PUFA consumption was associated with T2D even after adjustment for confounders, including other FA and BMI (HR 1.26; $95 \%$ CI 1.13, 1.41; upper tertile compared with lowest). Upon stratification by overweight $\left(\mathrm{BMI} \geq 25 \mathrm{~kg} / \mathrm{m}^{2}\right) /$ non-overweight, a positive association between total PUFA consumption and T2D was observed, but it was restricted to non-overweight women (HR 1.22; $95 \%$ CI 1.05, 1.42), whereas $n$-3 PUFA consumption was associated with increased T2D risk in both BMI strata (BMI $<25 \mathrm{~kg} / \mathrm{m}^{2}$ : HR 1.19; $95 \%$ CI 1.01, 1.40 and BMI $\geq 25 \mathrm{~kg} / \mathrm{m}^{2}:$ HR $1.38 ; 95 \%$ CI 1.20, 1.59). Within the $n-3$ PUFA, high DPA (HR 1.41; $95 \%$ CI 1.23, 1.63) and $\alpha$-linolenic acid (ALA) intakes were associated with increased T2D risk, but the effects of ALA were restricted to overweight women (HR 1.17; $95 \%$ CI 1.01, 1.36). Within the $n-6$ PUFA, only arachidonic acid (AA) intake was associated with T2D risk (HR 1.49; $95 \%$ CI 1.33, 1.66). The associations with DPA and AA persisted even after adjustment of their principal source in this cohort, the consumption of meat. The effects of PUFA are heterogeneous within the FA group. Intake of DPA and AA may contribute to T2D development.

\section{Key words: Fatty acids: Diabetes: $n$-3 PUFA: $n-6$ PUFA: Study cohorts: Dietary intakes: French women}

The incidence of type 2 diabetes (T2D) is influenced by many modifiable risk factors, such as overweight or obesity, physical inactivity, smoking and a poor diet, whose management could prevent $83-91 \%$ of cases in women ${ }^{(1,2)}$. One important risk factor is diet; however, focus has been placed heavily on carbohydrate consumption, although fats are responsible for a large part of energy intake and also have strong metabolic effects. Fatty acids (FA), the constituents of fat, are vital sources of energy and are required for normal growth and development ${ }^{(3)}$. Nevertheless, excessive intake of some FA may have negative health effects and their consumption should be limited $^{(4,5)}$
Current evidence concerning the association between FA consumption and the risk for T2D is sparse and controversial. Some studies have found no association between total fat intake and incidence of $\mathrm{T} 2 \mathrm{D}^{(6-8)}$; yet there is evidence to suggest that FA groups, and even individual FA within these groups, can have different effects on T2D risk ${ }^{(6-10)}$. Meta-analyses also suggest that the effects of $n-3$ PUFA intake differ by geographical region, with high intake associated with an increased risk for T2D in Western countries, no association in European countries and a decreased risk in Asian countries ${ }^{(11-13)}$. Moreover, $n-3$ and $n-6$ PUFA are almost always studied in their groups, and the effects of the individual PUFA rest scarcely

Abbreviations: AA, arachidonic acid; ALA, $\alpha$-linolenic acid; E3N, Etude Epidémiologique auprès des femmes de la Mutuelle Générale de l'Education Nationale; FA, fatty acid; HR, hazard ratio; T2D, type 2 diabetes; TFA, trans-unsaturated fatty acids.

* Corresponding author: G. Fagherazzi, fax +331421140 00, email guy.fagherazzi@gustaveroussy.fr 
examined despite the present $n-3$ PUFA health craze. In addition, most studies take the intake of other macronutrients into account when examining FA; however, many do not control for the consumption of the remaining FA, which is important, as groups and individual FA are never ingested in isolation and influence the metabolism of each other ${ }^{(14)}$.

The objective of this study was therefore to examine the associations between the main dietary FA intakes and the incidence of T2D. In particular, we focused on the relationship between $n-3$ and $n-6$ PUFA while adjusting for the concomitant intake of other FA.

\section{Methods}

\section{Study cohort}

E3N (Etude Epidémiologique auprès des femmes de la Mutuelle Générale de l'Education Nationale) is a prospective French cohort study of 98995 females born between 1925 and 1950 and initiated in 1990. It is the French constituent of the large European Prospective Investigation into Cancer and Nutrition (EPIC), and the EPIC sub-study devoted to diabetes: interaction of genetic and lifestyle factors on the incidence of type 2 diabetes (InterAct). Data are available from mailed questionnaires that participants have returned every $2-3$ years, in addition to a drugreimbursement claims database that has been available since 2004 from the participant's medical records (Mutuelle Générale de l'Education Nationale). The average response rate for each of the eleven questionnaire cycles has been $83 \%$, and to date the total loss to follow-up since 1990 has been 3\% (women who either never answered a questionnaire, withdrew from the study, or whose contact information was lost). All women signed letters of informed consent, in compliance with the French National Commission for Computerized Data and Individual Freedom. From the 98995 women in the cohort, women who did not complete the dietary questionnaire ( $n$ 24473), who had preexisting diabetes $(n$ 859), did not complete any questionnaires after the dietary questionnaire ( $n$ 890), or who had extreme values for the ratio between energy intake and required energy (i.e. the 1st and 99th percentiles of the distribution in the population) ( $n$ 1439) were excluded. Thus, the analysis included 71334 women, of whom 2610 had a validated T2D diagnosis during follow-up (June 1993-December 2011).

\section{Assessment of fatty acid consumption}

In June 1993, a validated 208-item dietary questionnaire was sent to participants to assess the habitual diet of the previous year. Food and drink consumption throughout the day took French meal patterns into account, as questions regarding each meal occasion, as well as snacks and appetisers, were also included. Divided into two sections, the first section of the questionnaire included habitual frequency and portion size questions, based on sixty-six food types and items grouped by meals. To quantify frequency, eleven categories were used: never or less than once per month; 1, 2 or 3 times/month; or 1-7 times/week. Portion sizes were indicated with the aid of a photo booklet. The second section had a detailed qualitative description of each food consumed from each food group and the frequency of its consumption (never; 1-3 times/month; or 1-7 times/week). After weighing the data from the first section by that from the second section, the average daily consumption of 208 types of foods and drinks was evaluated. The validity and reproducibility of the questionnaire have been previously described ${ }^{(15)}$.

FA consumption was then estimated using the database of food nutritional composition published by French Agency for Food, Environmental, and Occupational Health \& Safety. For each woman, intakes of the following principal FA were obtained: total SFA, total MUFA, total PUFA (including total $n-3$ PUFA and total $n$-6 PUFA), and total trans-unsaturated fatty acids (TFA) $(\mathrm{g} / \mathrm{d})$. In addition, we also estimated the consumptions of $n$-6 PUFA - linoleic acid (LA; 18:2n-6) and arachidonic acid (AA; $20: 4 n-6$ ) - and $n$-3 PUFA - $\alpha$-linolenic acid (ALA; $18: 3 n-3$ ), EPA (20:5n-3), DPA (22:5n-3) and DHA (22:6n-3).

\section{Ascertainment of diabetes}

Cases of T2D were first identified either in follow-up questionnaires (with declaration of at least one of the following: T2D; a self-reported, physician-recommended diabetic diet; use of diabetic medication; or hospitalisation due to diabetes) or they were identified as receiving diabetic drug reimbursements from health insurance records at least once between January 2004 and March 2012. Those identified through both methods were considered validated. All women were mailed a diabetesspecific questionnaire that included questions on the circumstances of the diagnosis (year, symptoms, biological exams, etc.), management (diabetic diet, physical activity, medications) and results of their most recent concentrations of fasting glucose and HbA1c. Cases were validated if one of the following was met: fasting plasma glucose $\geq 7.0 \mathrm{mmol} / 1$; random glucose $\geq 11.1 \mathrm{mmol} / 1$ at diagnosis; report of diabetic medication use; or last values of fasting glucose or HbA1c concentrations $\geq 7.0 \mathrm{mmol} / 1$ or $\geq 7 \%$, respectively. Prevalent and non-validated cases were excluded from analyses. Consequently, 2610 validated cases of incident diabetes and 68724 non-cases were analysed in the present study.

\section{Statistical analysis}

For time-independent variables with $<5 \%$ of values missing during follow-up, missing values were imputed with the median of the study population (quantitative variables) or the mode (qualitative variables). In the case of $\geq 5 \%$ of missing values, a 'missing' category was created. For time-dependent variables presenting with a missing value during follow-up, the value was imputed by the preceding provided value. If the missing value was the baseline value, it was imputed with the first available value.

Because of the high correlation between energy intake and nutrient intake, FA consumption was energy adjusted using the residual method to allow analyses independent of total energy intake $^{(16)}$. Each FA group was then divided into tertile groups of consumption, with women in the first tertile group of intake systematically employed as the reference category. Cox multivariable regression models with age as the timescale were used 
to estimate hazard ratios (HR) and 95\% CI of T2D risk. All variables were found to satisfy the proportional hazards assumption. The time at entry was the age at the start of followup and the exit time was the age when participants were diagnosed with diabetes, died, were lost to follow-up, or were censored at the end of the follow-up period, whichever occurred first. The univariate model included only the FA in question and was followed by model 1 , which was adjusted for daily energy intake $(\mathrm{kJ} / \mathrm{d}$ or $\mathrm{kcal} / \mathrm{d})$, alcohol consumption (grams of ethanol per $100 \mathrm{~g} / \mathrm{d}$ ) and non-dietary variables such as level of education (undergraduate $v$. postgraduate), smoking status (never $v$. current/former), hypertension (yes $v$. no), hypercholesterolaemia (yes $v$. no), family history of diabetes (yes $v$. no) and physical activity (metabolic equivalents (MET)h/week). Model 2 additionally adjusted for all types of FA besides the primary considered, allowing for the examination of the independent effects of specific FA subtypes. Finally, model 3 was adjusted for BMI $\left(<20,20-25,25-30\right.$ or $\left.>30 \mathrm{~kg} / \mathrm{m}^{2}\right)$.

In addition, stratification by BMI $\left(<25\right.$ and $\left.\geq 25 \mathrm{~kg} / \mathrm{m}^{2}\right)$ was performed to determine whether the effects of FA groups varied between overweight and non-overweight women. Models were adjusted in the same manner as in the analyses for the whole population, first for non-dietary variables, alcohol consumption and energy intake, and subsequently for other FA groups. All statistical analyses used SAS 9.2 software (PHREG procedure for Cox models; SAS Institute Inc.). All statistical tests were two-sided and considered significant at $P<0 \cdot 05$.

\section{Sensitivity analyses}

In order to test for potential reverse-causation, analyses were performed on a subpopulation excluding women who developed diabetes in the first 5 years after inclusion in the study. Individual $n-3$ and $n$ - 6 PUFA models were also adjusted for meat consumption, to account for residual confounding related to a major source of the FA, as it has already been reported in our cohort that a high intake of processed red meat was associated with an increased T2D risk ${ }^{(17)}$. Scores adhering to two dietary patterns, 'Mediterranean/Prudent' (characterised by a high diet quality, with a high consumption of fruit, vegetables, seafood, olive oil and sunflower oil) and 'Western' (characterised by a lower dietary quality, with a high consumption of meat, French fries, rice, pasta, potatoes, alcohol, butter, eggs, etc.), were also included in the models. These dietary quality scores were derived using principal component analysis and have been previously described in published work from our cohort ${ }^{(18)}$.

\section{Results}

\section{Baseline characteristics}

In total, 2610 cases of incident T2D were diagnosed during 1158131 person-years between June 1993 and December 2011. The incidence of T2D was 225/100000 person-years. Median follow-up was 18 years (ranging from $29 \mathrm{~d}$ to 18 years) for noncases and 11 years (ranging from $18 \mathrm{~d}$ to 18 years) for cases. Mean age of the study participants was 52.9 (SD 6.7) (Table 1). The majority $(66 \%)$ of women had a healthy BMI $\left(20-24.9 \mathrm{~kg} / \mathrm{m}^{2}\right)$, whereas $19.5 \%$ had an excess weight, including 3.3\% obese women. Hypertension was declared by $37 \cdot 1 \%$ of women and hypercholesterolaemia by $7 \cdot 1 \%$. In total, $11 \cdot 1 \%$ of women reported a family history of diabetes. The average daily energy intake was 8916 (sD 2276) kJ/d (2131 (sD 544) kcal/d) and the average physical activity was 49.4 (SD 50.5) MET-h/week. Mean FA group consumptions were as follows: 36.4 (SD 9.9) for SFA, 31.4 (SD 9.9) for MUFA, 1.6 (SD 0.7) for TFA, 14.2 (sD 5.4) for PUFA, 1.5 (SD 0.5 ) for $n$-3 PUFA, and 12.6 (sD 5.0) for $n-6$ PUFA g/d.

\section{SFA}

SFA consumption above the upper tertile was associated with an increased risk for T2D in the univariate analysis (HR 1.13; $95 \%$ CI 1.02, 1.24), and in the multivariable model adjusted for non-dietary factors, energy intake and alcohol (model 1: HR $1 \cdot 17 ; 95 \%$ CI 1.06, 1.28) (Table 2), when compared with the first tertile group. Further adjustment for other FA groups slightly decreased the association, rendering it non-significant (HR 1.13; $95 \%$ CI $0.97,1 \cdot 31$ ).

\section{MUFA}

MUFA consumption above the first tertile was associated with a higher risk for T2D in the unadjusted model and model 1 (third tertile group: HR 1.15; $95 \%$ CI 1.04, 1.27 and HR 1.32; $95 \%$ CI $1 \cdot 20,1 \cdot 46$, respectively). Additional adjustment for FA groups in model 2 decreased the strength of the association in the second tertile group (HR 1.10; 95\% CI 0.99, 1.22), whereas the association in the third tertile group became statistically non-significant after adjustment for BMI (model 3: HR 1.06; 95\% CI 0.95, 1.19).

PUFA

PUFA intake was associated with a $29 \%$ increased risk for T2D in women above the third tertile of intake after adjustment for other types of FA in model 2 (HR 1.29; $95 \%$ CI 1.17, 1.43). However, there was no longer an association after adjusting for BMI (model 3: HR 1.06; $95 \%$ CI 0.96, 1.17). Positive associations were observed for both $n$ - 3 and $n$ - 6 PUFA in model 1 , but after adjusting for other FA and BMI only the association above the third tertile of $n$ - 3 PUFA intake remained (HR 1.26; $95 \%$ CI 1.13, 1.41).

\section{Trans-unsaturated fatty acids}

The second tertile group of TFA intake was associated with a lower T2D risk in the univariate analysis (HR 0.87; $95 \% \mathrm{CI} 0.79,0.96)$ and in model 2 (HR 0.88; 95\% CI 0.78, 0.98), adjusted for other FA, but not in model 1 (HR 0.93; 95\% CI 0.85, 1.02). After adjustment for BMI, high TFA intake showed a borderline statistically significant association with T2D (HR 0.90; 95\% CI 0.80, 1.00).

\section{Stratification by $B M I$}

After stratification by BMI, in the fully adjusted models, neither SFA nor MUFA nor TFA were associated with T2D risk in either BMI stratum (Fig. 1, online Supplementary Table S1). Regarding total PUFA, a high intake was associated with increased T2D 
Table 1. Baseline characteristics of the study population (E3N (Etude Epidémiologique auprès des femmes de la Mutuelle Générale de l'Education Nationale) cohort data; $n 71334$ women)

(Mean values and standard deviations; numbers and percentages)

\begin{tabular}{|c|c|c|c|c|c|c|}
\hline Variables & $\begin{array}{l}\text { Study population } \\
\quad(n 71334)\end{array}$ & SD or $(\%)$ & $\begin{array}{l}\text { Diabetes non-cases } \\
\quad(n 68724)\end{array}$ & SD or $(\%)$ & $\begin{array}{l}\text { Incident diabetes } \\
\text { cases }(n \text { 2610) }\end{array}$ & SD or $(\%)$ \\
\hline Age (years) & $52 \cdot 9$ & $6 \cdot 7$ & 5.8 & $6 \cdot 7$ & 54.4 & $6 \cdot 7$ \\
\hline \multicolumn{7}{|l|}{$\operatorname{BMI}\left(\mathrm{kg} / \mathrm{m}^{2}\right)(n(\%))$} \\
\hline$<20$ & 10307 & (14.5) & 10239 & $(14.9)$ & 68 & $(2 \cdot 6)$ \\
\hline $20-24 \cdot 9$ & 47101 & $(66 \cdot 0)$ & 46073 & $(67 \cdot 0)$ & 1028 & $(39.4)$ \\
\hline $25-30$ & 11554 & $(16 \cdot 2)$ & 10572 & $(15.4)$ & 982 & $(37.6)$ \\
\hline$>30$ & 2372 & (3.3) & 1840 & $(2 \cdot 7)$ & 532 & $(20.4)$ \\
\hline \multicolumn{7}{|l|}{ Birth cohort $(n(\%))$} \\
\hline Before 1930 & 5437 & $(7 \cdot 6)$ & 5186 & $(7 \cdot 6)$ & 251 & $(9.6)$ \\
\hline $1930-1934$ & 9175 & $(12 \cdot 9)$ & 8702 & $(12 \cdot 7)$ & 473 & $(18 \cdot 1)$ \\
\hline 1935-1939 & 13530 & (19.0) & 12991 & (18.9) & 539 & $(20.7)$ \\
\hline 1940-1944 & 17142 & $(24.0)$ & 16494 & $(24.0)$ & 648 & $(24 \cdot 8)$ \\
\hline After 1944 & 26050 & $(36.5)$ & 25351 & $(36.9)$ & 699 & $(26 \cdot 8)$ \\
\hline \multicolumn{7}{|l|}{ Education $(n(\%))$} \\
\hline Undergraduate & 7913 & $(11 \cdot 1)$ & 7493 & $(10 \cdot 9)$ & 420 & $(16 \cdot 1)$ \\
\hline Graduate/postgraduate & 63421 & (88.9) & 61231 & $(89 \cdot 1)$ & 2190 & (83.9) \\
\hline \multicolumn{7}{|l|}{ Smoking status $(n(\%))$} \\
\hline Never & 38504 & $(54 \cdot 0)$ & 37115 & $(54.0)$ & 1389 & $(53.2)$ \\
\hline Current/former & 32830 & $(46 \cdot 0)$ & 31609 & $(46 \cdot 0)$ & 1221 & $(46 \cdot 8)$ \\
\hline \multicolumn{7}{|l|}{ Hypertension ( $n(\%))$} \\
\hline No & 44857 & $(62.9)$ & 43773 & $(63 \cdot 7)$ & 1084 & $(41.5)$ \\
\hline Yes & 26477 & $(37 \cdot 1)$ & 24951 & $(36 \cdot 3)$ & 1526 & $(58.5)$ \\
\hline \multicolumn{7}{|l|}{ Hypercholesterolaemia treatment $(n(\%))$} \\
\hline No & 66297 & (92.9) & 64085 & (93.3) & 2212 & $(3 \cdot 1)$ \\
\hline Yes & 5037 & $(7 \cdot 1)$ & 4639 & $(6 \cdot 8)$ & 398 & $(15 \cdot 3)$ \\
\hline \multicolumn{7}{|l|}{ Family history of T2D ( $n(\%))$} \\
\hline No & 63432 & $(88.9)$ & 61451 & $(89.4)$ & 1981 & $(75 \cdot 9)$ \\
\hline Yes & 7902 & $(11 \cdot 1)$ & 7273 & $(10 \cdot 6)$ & 629 & $(24 \cdot 1)$ \\
\hline Physical activity (MET-h/week) & 49.4 & 50.5 & $49 \cdot 3$ & $50 \cdot 1$ & $50 \cdot 0$ & $59 \cdot 2$ \\
\hline Alcohol consumption ( $\mathrm{g}$ of ethanol per $100 \mathrm{~g} / \mathrm{d}$ ) & 11.6 & 13.9 & 11.6 & $13 \cdot 8$ & $12 \cdot 1$ & $15 \cdot 9$ \\
\hline Daily energy intake $(\mathrm{kJ} / \mathrm{d})$ & 8914.4 & 2274.8 & $8906 \cdot 8$ & $2266 \cdot 1$ & $9114 \cdot 8$ & 2481.5 \\
\hline Daily energy intake (kcal/d) & $2130 \cdot 6$ & 543.7 & 2128.8 & 541.6 & 2178.5 & $593 \cdot 1$ \\
\hline \multicolumn{7}{|l|}{ Average consumption (g/d) } \\
\hline SFA & $36 \cdot 4$ & $13 \cdot 0$ & $36 \cdot 4$ & $12 \cdot 9$ & $37 \cdot 6$ & $14 \cdot 1$ \\
\hline MUFA & 31.3 & 9.9 & 31.3 & $9 \cdot 8$ & $32 \cdot 6$ & $10 \cdot 8$ \\
\hline TFA & 1.6 & 0.7 & 1.6 & 0.7 & 1.6 & 0.7 \\
\hline PUFA & 14.2 & $5 \cdot 3$ & $14 \cdot 1$ & $5 \cdot 3$ & $15 \cdot 2$ & 5.9 \\
\hline$n-3$ PUFA & 1.5 & 0.5 & 1.5 & 0.5 & 1.6 & 0.6 \\
\hline$n-6$ PUFA & $12 \cdot 6$ & $5 \cdot 0$ & $12 \cdot 6$ & 5.0 & $13 \cdot 6$ & $5 \cdot 6$ \\
\hline
\end{tabular}

T2D, type 2 diabetes; MET, metabolic equivalents; TFA, trans-unsaturated fatty acids.

risk in non-overweight women (HR 1.22; $95 \%$ CI 1.05, 1.42), but not in overweight women (HR 1.02; $95 \%$ CI 0.90, 1.16). n-6 PUFA were not associated with T2D risk in either BMI stratum (HR 1.13; $95 \%$ CI 0.96, 1.32 and HR 0.95; $95 \%$ CI 0.83, 1.08, respectively, for non-overweight and overweight women), whereas high $n$-3 PUFA consumption was associated with an increased risk for T2D in both strata in fully adjusted models (HR 1.19; 95\% CI 1.01, 1.40 and HR 1.38; 95\% CI 1.20, 1.59, respectively, for non-overweight and overweight women).

\section{n-6 Fatty acids}

LA was not significantly associated with T2D risk in the whole population (HR $0.97 ; 0.87,1.07$ ) or in either BMI stratum (Table 3). Conversely, a high consumption of AA was associated with an almost $50 \%$ increased risk for T2D (HR 1.49; $95 \%$ CI 1.33, 1.66). Within BMI strata, AA intake above the third tertile was associated with T2D risk in non-overweight women (HR 1.50; $95 \%$ CI 1.28, 1.76) and intake above the second tertile was associated with T2D risk in overweight women (HR 1.74; $95 \%$ CI $1.49,2 \cdot 03)$.

\section{n-3 Fatty acids}

In the full population, neither ALA intake nor intake of longchain $n$-3 PUFA (EPA or DHA) was associated with T2D in the fully adjusted models (HR 1.03; 95\% CI 0.92, 1.15; HR 0.88; $95 \%$ CI $0.67,1.15$; HR $1.11 ; 95 \%$ CI $0.85,1.44$, respectively). However, high DPA intake above the second tertile was associated with an increased risk for T2D (HR 1.41; 95\% CI 1.23, 1.63). There was no association with EPA or DHA in models stratified on BMI. However, high ALA intake was associated with an increased T2D risk in women with excess weight (HR 1.17; 95\% CI 1.01, 1.36) for the highest tertile group, but not in the normal-weight women (HR 0.90; $95 \%$ CI 0.75, 1.07). High DPA intake was associated with an increased risk for T2D in both BMI strata (HR 1.45; $95 \%$ CI 1.17, 1.80 and HR 1.54; $95 \%$ CI $1 \cdot 27,1 \cdot 85$, respectively). 
Table 2. Cox proportional hazards ratios for the risk for incident type 2 diabetes by fatty acid consumption $(\mathrm{g} / \mathrm{d})$ in the E3N (Etude Epidémiologique auprès des femmes de la Mutuelle Générale de l'Education Nationale) study cohort ( $n 71334$ women)(Hazard ratios (HR) and $95 \%$ confidence intervals)

\begin{tabular}{|c|c|c|c|c|c|c|c|c|c|}
\hline \multirow{2}{*}{$\begin{array}{l}\text { Variables } \\
(\mathrm{g} / \mathrm{d})\end{array}$} & \multirow{2}{*}{$\begin{array}{c}\text { No. of cases/ } \\
\text { non-cases }\end{array}$} & \multicolumn{2}{|c|}{ Univariate } & \multicolumn{2}{|c|}{ Model $1^{*}$} & \multicolumn{2}{|c|}{ Model 2† } & \multicolumn{2}{|c|}{ Model 3‡ } \\
\hline & & $\mathrm{HR}$ & $95 \% \mathrm{Cl}$ & HR & $95 \% \mathrm{Cl}$ & $\mathrm{HR}$ & $95 \% \mathrm{Cl}$ & $\mathrm{HR}$ & $95 \% \mathrm{Cl}$ \\
\hline \multicolumn{10}{|l|}{ SFA } \\
\hline$<33.3$ & $843 / 22698$ & \multicolumn{2}{|c|}{1 (Ref.) } & \multicolumn{2}{|c|}{1 (Ref.) } & \multicolumn{2}{|c|}{1 (Ref.) } & \multicolumn{2}{|c|}{1 (Ref.) } \\
\hline $33 \cdot 3-38 \cdot 9$ & $847 / 22692$ & 1.04 & $0.95,1.14$ & $1 \cdot 10$ & $1.00,1.21$ & $1 \cdot 11$ & $0.99,1.25$ & 1.06 & $0.95,1.19$ \\
\hline$\geq 38.9$ & $920 / 23334$ & 1.13 & $1.02,1.24$ & 1.17 & $1.06,1.28$ & 1.13 & $0.97,1.31$ & 1.07 & $0.92,1.25$ \\
\hline $\begin{array}{l}P_{\text {for trend }} \\
\text { MUFA }\end{array}$ & & \multicolumn{2}{|c|}{0.01} & \multicolumn{2}{|c|}{0.001} & \multicolumn{2}{|c|}{0.14} & \multicolumn{2}{|c|}{0.40} \\
\hline$<28.7$ & $785 / 22755$ & \multicolumn{2}{|c|}{1 (Ref.) } & \multicolumn{2}{|c|}{1 (Ref.) } & \multicolumn{2}{|c|}{1 (Ref.) } & \multicolumn{2}{|c|}{1 (Ref.) } \\
\hline $28 \cdot 7-33 \cdot 3$ & $839 / 22702$ & $1 \cdot 11$ & $1.01,1.23$ & 1.15 & $1.04,1.27$ & $1 \cdot 10$ & $0.99,1.22$ & 1.01 & $0.91,1.12$ \\
\hline$\geq 33.3$ & $986 / 23267$ & 1.31 & $1.19,1.44$ & 1.32 & $1.20,1.46$ & 1.23 & $1.10,1.38$ & 1.06 & $0.95,1.19$ \\
\hline $\begin{array}{l}P_{\text {for trend }} \\
\text { PUFA }\end{array}$ & & \multicolumn{2}{|c|}{$<0.0001$} & \multicolumn{2}{|c|}{$<0.0001$} & \multicolumn{2}{|c|}{$<0.001$} & \multicolumn{2}{|c|}{0.25} \\
\hline$<12 \cdot 0$ & $720 / 22821$ & \multicolumn{2}{|c|}{1 (Ref.) } & \multicolumn{2}{|c|}{1 (Ref.) } & \multicolumn{2}{|c|}{1 (Ref.) } & \multicolumn{2}{|c|}{1 (Ref.) } \\
\hline $12 \cdot 0-15 \cdot 3$ & $835 / 22704$ & $1 \cdot 17$ & $1.06,1.29$ & 1.14 & $1.03,1.26$ & $1 \cdot 13$ & $1.02,1.25$ & 1.03 & $0.93,1.14$ \\
\hline$\geq 15 \cdot 3$ & $1055 / 23199$ & 1.46 & $1.33,1.61$ & 1.34 & $1.21,1.47$ & 1.29 & $1.17,1.43$ & 1.06 & $0.96,1.17$ \\
\hline $\begin{array}{l}\bar{P}_{\text {for trend }} \\
n-6 \text { PUFA }\end{array}$ & & \multicolumn{2}{|c|}{$<0.0001$} & \multicolumn{2}{|c|}{$<0.0001$} & \multicolumn{2}{|c|}{$<0.0001$} & \multicolumn{2}{|c|}{0.24} \\
\hline$<10.5$ & $734 / 22807$ & & & & & & & & \\
\hline $10 \cdot 5-13.7$ & $840 / 22700$ & 1.15 & $1.04,1.27$ & 1.13 & $1.02,1.25$ & 1.07 & $0.96,1.18$ & 1.01 & $0.91,1.12$ \\
\hline$\geq 13.7$ & $1036 / 23217$ & 1.41 & $1.28,1.55$ & 1.29 & $1.17,1.42$ & $1 \cdot 15$ & $1.04,1.27$ & 1.00 & $0.90,1.10$ \\
\hline $\begin{array}{l}P_{\text {for trend }} \\
n-3 \text { PUFA }\end{array}$ & & & & & & & & & \\
\hline$<1 \cdot 3$ & $665 / 22876$ & & & & & & & & \\
\hline $1 \cdot 3-1 \cdot 6$ & $819 / 22720$ & 1.25 & $1.13,1.38$ & 1.23 & $1.11,1.37$ & $1 \cdot 18$ & $1.06,1.31$ & $1 \cdot 10$ & $0.99,1.22$ \\
\hline$\geq 1.6$ & $1126 / 23128$ & 1.68 & $1.52,1.84$ & 1.60 & $1.45,1.76$ & 1.47 & $1.32,1.64$ & 1.26 & $1.13,1.41$ \\
\hline $\begin{array}{l}\bar{P}_{\text {for trend }} \\
\text { TFA }\end{array}$ & & & & & & & & & \\
\hline$<1.4$ & $920 / 22621$ & & & & & & & & \\
\hline $1.4-1.7$ & $794 / 22745$ & 0.87 & $0.79,0.96$ & 0.93 & $0.85,1.02$ & 0.88 & $0.78,0.98$ & 0.90 & $0.80,1.00$ \\
\hline$\geq 1 \cdot 7$ & $896 / 23358$ & 0.98 & $0.89,1.07$ & 1.03 & $0.94,1.13$ & 0.93 & $0.81,1.07$ & 1.02 & $0.89,1.17$ \\
\hline $\bar{P}_{\text {for trend }}$ & & & & & & & & & \\
\hline
\end{tabular}

Ref., referent values; TFA, trans-unsaturated fatty acids.

* Model 1: adjusted for daily energy intake, alcohol consumption and non-dietary factors such as level of education, family history of diabetes, physical activity, hypertension, hypercholesterolaemia and smoking status.

† Model 2: model $1+$ tertile groups of remaining fatty acid groups.

$\ddagger$ Model 3: model 2+BMI.
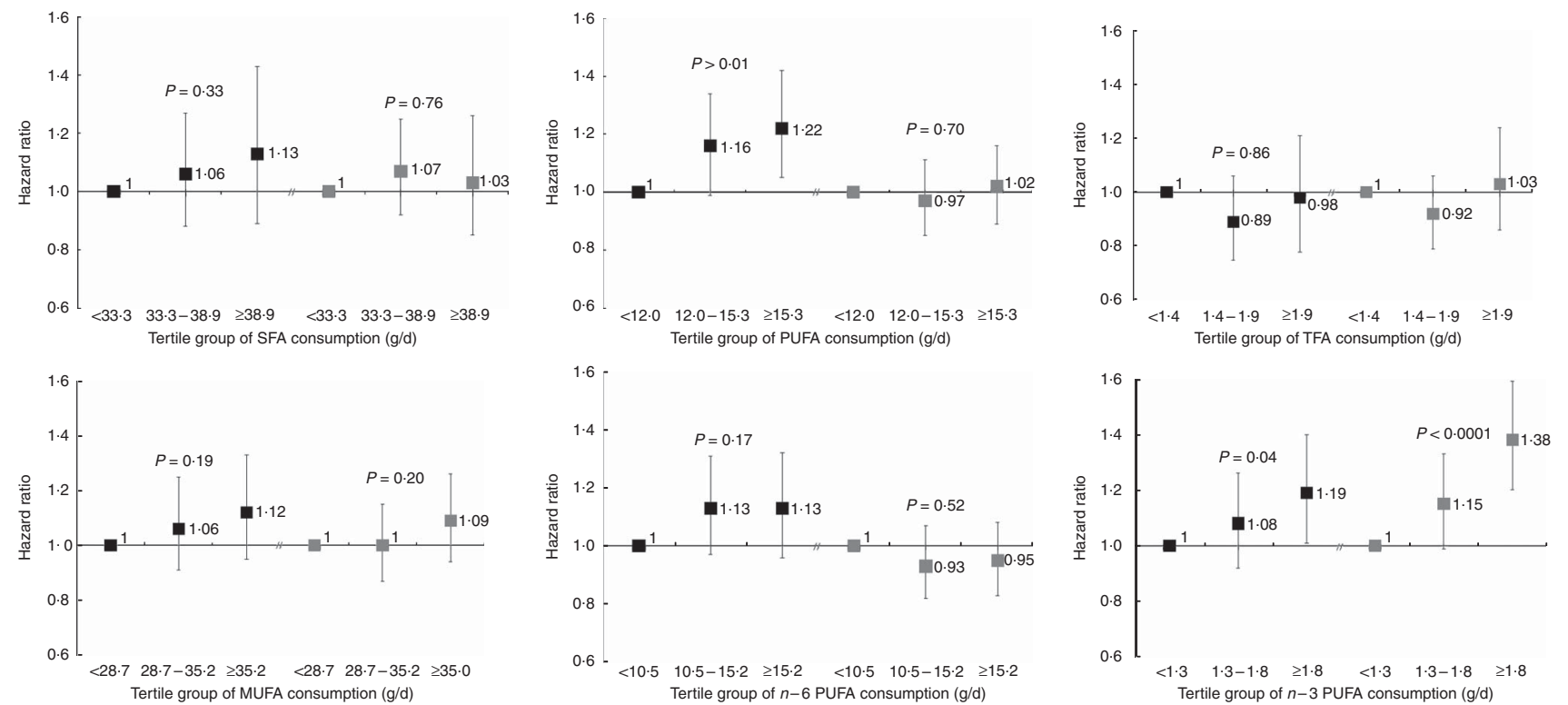

Fig. 1. BMl-stratified Cox proportional hazard ratios $(95 \% \mathrm{Cl}$ ) of type 2 diabetes by tertile group of fatty acid consumption ( $\mathrm{g} / \mathrm{d})$. Models adjusted for daily energy intake, alcohol consumption, level of education, family history of diabetes, physical activity, hypertension, hypercholesterolaemia, smoking status and tertile groups of remaining fatty acid groups. TFA, trans-unsaturated fatty acids. $\square, \mathrm{BMl}<25 \mathrm{~kg} / \mathrm{m}^{2} ; \square, \mathrm{BMI} \geq 25 \mathrm{~kg} / \mathrm{m}^{2}$. 
Table 3. Cox proportional hazards ratios of the risk for incident type 2 diabetes by $n-3$ and $n-6$ PUFA consumption ( $/$ /d) with BMI stratification in the E3N (Etude Epidémiologique auprès des femmes de la Mutuelle Générale de l'Education Nationale) study cohort ( $n$ 71334)

(Hazard ratios (HR) and $95 \%$ confidence intervals)

\begin{tabular}{|c|c|c|c|c|c|c|c|c|c|}
\hline \multirow[b]{2}{*}{ Variables $(\mathrm{g} / \mathrm{d})$} & \multirow{2}{*}{$\begin{array}{c}\text { No. of cases/ } \\
\text { non-cases }\end{array}$} & \multicolumn{2}{|c|}{$n 71334$} & \multirow{2}{*}{$\begin{array}{c}\text { No. of cases/ } \\
\text { non-cases }\end{array}$} & \multicolumn{2}{|c|}{$\mathrm{BMI}<25 \mathrm{~kg} / \mathrm{m}^{2}(n 57408)$} & \multirow{2}{*}{$\begin{array}{c}\text { No. of cases/ } \\
\text { non-cases }\end{array}$} & \multicolumn{2}{|c|}{$\mathrm{BMI} \geq 25 \mathrm{~kg} / \mathrm{m}^{2}(n$ 13926) } \\
\hline & & $\mathrm{HR}$ & $95 \% \mathrm{Cl}$ & & HR & $95 \% \mathrm{Cl}$ & & $\mathrm{HR}$ & $95 \% \mathrm{Cl}$ \\
\hline \multicolumn{10}{|l|}{ ALA } \\
\hline$<0.90$ & 757/22 784 & \multicolumn{2}{|c|}{1 (Ref.) } & $358 / 19213$ & \multicolumn{2}{|c|}{1 (Ref.) } & $399 / 3571$ & \multicolumn{2}{|c|}{1 (Ref.) } \\
\hline $0.90-1 \cdot 14$ & $843 / 22696$ & 1.00 & $0.90,1.12$ & $365 / 18649$ & 0.93 & $0.79,1.09$ & $478 / 4047$ & 1.08 & $0.94,1.25$ \\
\hline$\geq 1 \cdot 14$ & 1010/23244 & 1.03 & $0.92,1.15$ & $373 / 18450$ & 0.90 & $0.75,1.07$ & $637 / 4794$ & $1 \cdot 17$ & $1.01,1.36$ \\
\hline $\begin{array}{l}\bar{P}_{\text {for trend }} \\
\text { EPA }\end{array}$ & & \multicolumn{2}{|r|}{0.62} & & \multicolumn{2}{|c|}{0.24} & & \multicolumn{2}{|c|}{0.04} \\
\hline$<0.09$ & $711 / 22830$ & \multicolumn{2}{|c|}{1 (Ref.) } & $329 / 19275$ & \multicolumn{2}{|c|}{1 (Ref.) } & $382 / 3555$ & \multicolumn{2}{|c|}{1 (Ref.) } \\
\hline $0.09-0.20$ & $823 / 22716$ & 0.88 & $0.73,1.06$ & $344 / 18694$ & 0.86 & $0.65,1.14$ & $479 / 4022$ & 0.87 & $0.67,1.12$ \\
\hline$\geq 0 \cdot 20$ & 1076/23178 & 0.88 & $0.67,1.15$ & $423 / 18343$ & 0.79 & $0.53,1.19$ & $653 / 4835$ & 0.93 & $0.65,1.32$ \\
\hline $\begin{array}{l}P_{\text {for trend }} \\
\text { DPA }\end{array}$ & & \multicolumn{2}{|r|}{0.51} & & \multicolumn{2}{|c|}{0.32} & & \multicolumn{2}{|c|}{0.96} \\
\hline$<0.05$ & $610 / 22931$ & \multicolumn{2}{|c|}{1 (Ref.) } & 307/19912 & \multicolumn{2}{|c|}{1 (Ref.) } & $303 / 3019$ & \multicolumn{2}{|c|}{1 (Ref.) } \\
\hline $0.05-0.08$ & $805 / 22734$ & $1 \cdot 15$ & $1.02,1.30$ & $364 / 18723$ & $1 \cdot 23$ & $1.04,1.47$ & $441 / 4011$ & $1 \cdot 15$ & $0.98,1.36$ \\
\hline$\geq 0.08$ & $1195 / 23059$ & \multirow{2}{*}{\multicolumn{2}{|c|}{$<0.0001$}} & $425 / 17677$ & 1.45 & $1 \cdot 17,1 \cdot 80$ & $770 / 5382$ & 1.54 & $1 \cdot 27,1.85$ \\
\hline $\begin{array}{l}P_{\text {for trend }} \\
\text { DHA }\end{array}$ & & & & & \multicolumn{2}{|c|}{$<0.01$} & & \multicolumn{2}{|c|}{$<0.0001$} \\
\hline$<0.19$ & $702 / 22839$ & \multicolumn{2}{|c|}{1 (Ref.) } & $327 / 19243$ & \multicolumn{2}{|c|}{1 (Ref.) } & $375 / 3596$ & \multicolumn{2}{|c|}{1 (Ref.) } \\
\hline $0.19-0.38$ & $839 / 22701$ & $1 \cdot 15$ & $0.95,1.38$ & $344 / 18743$ & 1.06 & $0.80,1.41$ & $495 / 3958$ & 1.23 & $0.96,1.58$ \\
\hline$\geq 0.38$ & $1069 / 23184$ & \multirow{2}{*}{\multicolumn{2}{|c|}{$\begin{array}{ll}1.11 & 0.65,1.44 \\
0.65\end{array}$}} & $425 / 18326$ & $1 \cdot 24$ & $0.83,1.86$ & $644 / 4858$ & 1.01 & $0.71,1.43$ \\
\hline $\bar{P}_{\text {for trend }}$ & & & & & & & & & \\
\hline LA & & & & & & & & & \\
\hline$<10.3$ & $740 / 22801$ & & (Ref.) & $330 / 19348$ & & ef.) & $410 / 3453$ & & ef.) \\
\hline $10 \cdot 3-13 \cdot 5$ & 838/22 702 & 0.98 & $0.89,1.08$ & $382 / 18764$ & $1 \cdot 10$ & $0.94,1.28$ & $456 / 3938$ & 0.90 & $0.78,1.03$ \\
\hline$\geq 13.5$ & $1032 / 23221$ & 0.97 & $0.87,1.07$ & $384 / 18200$ & 1.08 & $0.92,1.26$ & $648 / 5021$ & 0.91 & $0.80,1.04$ \\
\hline AA $P_{\text {for trend }}$ & & & 0.52 & & & & & & \\
\hline$<0.19$ & $579 / 22961$ & & (Ref.) & $327 / 20068$ & & ef.) & $252 / 2893$ & & ef.) \\
\hline $0.19-0.25$ & $750 / 22790$ & $1 \cdot 11$ & $0.99,1.24$ & $329 / 18869$ & 1.05 & $0.90,1.23$ & $421 / 3921$ & 1.28 & $1.09,1.50$ \\
\hline$\geq 0.25$ & $1281 / 22973$ & 1.49 & $1.33,1.66$ & $440 / 17375$ & 1.50 & $1.28,1.76$ & $841 / 5598$ & 1.74 & $1.49,2.03$ \\
\hline$P_{\text {for trend }}$ & & & .0001 & & & 001 & & & 001 \\
\hline
\end{tabular}

ALA, $a$-linolenic acid (18:3n-3); Ref., referent values; EPA, 20:5n-3; DPA, 22:5n-3; DHA, 22:6n-3; LA, linoleic acid (18:2n-6); AA, arachidonic acid (20:4n-6).

\section{Sensitivity analysis}

In a sensitivity analysis excluding the 388 women who developed diabetes during the first 5 years of follow-up, thus on a population of 70946 women with 2222 incident cases of diabetes, the associations were similar to those in the full analysis (online Supplementary Table S2).

Meat was the food group that contributed most to both AA and DPA intakes ( 42.7 and $31.3 \%$, respectively). Dietary ALA primarily came from dairy products $(11.9 \%)$, followed by fats $(8.2 \%)$, olives $(7.0 \%)$, and meat, processed meats and offal (6.9\%). After additional adjustment for total meat consumption, including processed meats and offal, ALA intake was no longer associated with T2D risk (HR 1.03; $95 \%$ CI 0.92, 1.15) (online Supplementary Table S3), whereas high intakes of DPA and AA remained associated with T2D risk (HR 1.21; $95 \%$ CI 1.03, 1.42 and HR 1.40; 95\% CI 1.23, 1.59, respectively). After stratification by BMI, high DPA intake was associated with T2D risk only in women with $\mathrm{BMI} \geq 25 \mathrm{~kg} / \mathrm{m}^{2}$ (HR 1.33; $95 \%$ CI 1.08, 1.64), whereas high AA intake increased T2D risk in the nonoverweight strata (HR 1.31; 95\% CI 1.09, 1.59) and all intakes above the reference tertile group increased T2D risk in the overweight BMI strata (HR 1.68; $95 \%$ CI 1.41, 2.00, third tertile group compared with first tertile group).
Adjustment for the overall dietary pattern using scores adhering to 'Mediterranean/Prudent' or 'Western' dietary patterns yielded similar results, with the exception of a modest increase in risk for high TFA intake (results not shown).

\section{Discussion}

In this prospective cohort of middle-aged women, high total $n-3$ PUFA intake was associated with T2D risk, irrespective of the BMI. Yet, within the $n-3$ PUFA, only DPA and ALA (ALA in overweight women only) were associated with increased T2D risk. Total $n-6$ PUFA intake was not associated with T2D risk, but AA intake was associated with increased T2D risk. The other groups of FA (SFA, MUFA, TFA) were not associated with T2D risk.

\section{SFA}

The absence of an association between SFA intake and T2D risk has already been reported ${ }^{(7,8,19-21)}$. However, biomarkers of very long-chain SFA and odd-chain SFA have been associated with decreased T2D risk, and even-chain SFA with increased risk, either individually or combined ${ }^{(10,21,22)}$. Biomarkers of odd-chain SFA are considered representative of dietary 
intake $^{(10)}$, whereas even-chain SFA are also endogenously produced $^{(23)}$. It can thus be suggested that the absence of an association in our study could be due to heterogeneous effects within SFA; yet this phenomenon should also be estimated using dietary intake of SFA.

\section{MUFA}

Our findings that MUFA intake was not associated with incident T2D are in agreement with other studies ${ }^{(7,8,21)}$. Yet, some studies have found extra virgin olive oil, an important source of MUFA $^{(24)}$, to be beneficial for T2D risk ${ }^{(25,26)}$. However, in our cohort, dairy and meat intakes are the primary sources of MUFA ( 13.4 and $11.9 \%$, respectively) and prospective studies suggest either an inverse or no association of dairy intake with T2D risk $^{(27,28)}$ and a negative association of meat intake with T2D risk $^{(17,28)}$, offering an explanation why we observed no association.

\section{PUFA}

Our findings contrast other findings in which PUFA intake was associated with decreased T2D risk ${ }^{(7,8)}$. However, PUFA are a group of FA likely to have different effects on T2D risk. Our results support this hypothesis as individual FA within the $n-3$ and $n$ - 6 PUFA groups had differing effects on T2D risk.

To our knowledge, only an Australian case-cohort study examined the relationship between the dietary intake of individual $n-6$ PUFA and T2D and found no association with any of the $n-6$ PUFA $^{(29)}$. Contrary to us, studies using plasma measurements of $n$ - 6 PUFA reported no association with AA, and have mixed results concerning LA, as one found no association and two reported an inverse association with T2D ${ }^{(19,21,29)}$

Few studies have examined the associations of individual $n-3$ PUFA on T2D risk; yet, available evidence concerning the dietary intake of ALA contradicts our results and suggests the absence of an association with T2D risk ${ }^{(11,13,29)}$. However, ALA may be differentially associated with T2D in populations, with a decreased risk in Asian countries but no association in Western countries $^{(11)}$. The marine $n$ - 3 PUFA (EPA, DPA and DHA) are often grouped together and rarely analysed on their own. However, the Australian case-cohort study did look at each individually and did not find any associations with T2D risk ${ }^{(29)}$. But average intakes of DPA and AA were lower in their population than in the $\mathrm{E} 3 \mathrm{~N}$ cohort $(0.03$ (SD 0.03$)$ and 0.07 $(\mathrm{SD} 0.03) \mathrm{g} / \mathrm{d}$, respectively, for DPA and 0.04 (SD 0.03) and 0.22 $(\mathrm{sD} 0.09) \mathrm{g} / \mathrm{d}$, respectively, for $\mathrm{AA})^{(29)}$. In addition, although meat is the primary contributor to AA intake in the Australian population $(70.2 \%)$, fish and meat provided nearly equal contributions to the consumption of DPA ( 50.4 and $49.2 \%$, respectively $)^{(30)}$. In meta-analyses stratified by sex, intake of marine $n$-3 PUFA was not associated with T2D risk in the whole population or in men, but was associated with an increased T2D risk in women ${ }^{(12)}$. As the Australian case-cohort differed in the amounts and dietary sources of certain PUFA and included both men and women, this could explain the discrepancies with our findings.

\section{Trans-unsaturated fatty acids}

Total dietary intake of TFA was not associated with incident T2D, supporting the findings of other prospective studies ${ }^{(8,19,31)}$ and contrasting with one study ${ }^{(7)}$. Residual dietary confounding could explain the inconsistency in results, as the mentioned cohorts all found significant associations with TFA until adjustment for other dietary factors ${ }^{(8,19,31)}$, or TFA may also be a heterogeneous group. Plasma measurements of two TFA have been associated with an increased T2D risk ${ }^{(31)}$, while evidence from a prospective cohort suggests that trans-palmitoleate (trans-16:1n-7), a naturally occurring dairy TFA, is associated with decreased T2D risk ${ }^{(32)}$

\section{Potential biological pathways}

One of the mechanisms through which FA are believed to affect the development of T2D is through their effects on cell membranes. The FA tails of the phospholipid (PL) bilayer can differ in both length and saturation (with double bonds reducing their ability to pack closely together); thus the FA composition of the cell membrane affects its fluidity, membrane protein incorporation, enzyme activities and receptor functions ${ }^{(33)}$. As recent studies have demonstrated that the FA composition of the membrane is at least partially determined by the diet, this may have important health implications ${ }^{(34,35)}$. Participants randomly assigned to a diet with a high proportion of SFA later showed higher proportions of SFA in skeletal muscle PL, compared with individuals consuming a high-proportion MUFA diet ${ }^{(34)}$. Increasing ratios of SFA:PUFA in the skeletal muscle cell membrane have been observed to decrease glucose effectiveness and insulin sensitivity in humans, which could theoretically lead to $\mathrm{T} 2 \mathrm{D}^{(36,37)}$. However, both the proportion and absolute amount of FA can affect plasma concentrations, and thus both may be important in determining skeletal muscle PL composition as well, and could explain why we did not observe a similar relationship with SFA in our study ${ }^{(35)}$. In addition, as the food preparation methods could alter the FA content differentially by FA group and type of food, this offers another theory to the discrepancy in our results with the biological studies ${ }^{(38)}$.

FA may also exert their effects through gene expression, as they act as signalling molecules of genes coding for metabolic enzymes ${ }^{(39)}$. Studies have shown that persistent exposure of pancreatic islet cells to high levels of free FA prevented transcription of acetyl-CoA carboxylase, an enzyme required for glucose-stimulated insulin release from the pancreas ${ }^{(39)}$. A few studies have also suggested that AA could stimulate insulin secretion $^{(40,41)}$ and suggested that the plasma concentration of AA could be a key determinant of insulin resistance ${ }^{(42)}$. However, the relationship between AA and insulin is not yet clear, as studies also suggest that in the case of diabetes, insulin may activate enzymes responsible for AA synthesis ${ }^{(43)}$. Pure DPA has been difficult to isolate and was not readily accessible; hence, there is no literature on the biological relationship between DPA and T2D ${ }^{(44)}$.

\section{Strengths and limitations}

Potential limitations should be considered. The dietary estimates are based on a single questionnaire at baseline; thus, 
misclassification of exposure is possible. However, as the study is prospective, any effects are likely to be non-differential and would lead to an attenuation of the true association. T2D cases were carefully identified and validated; yet it is possible there was an under-ascertainment of T2D cases, especially in asymptomatic women. With our large sample size, small deviations could lead to small $P$-values when comparing the characteristics between cases and non-cases, but these would not affect the $P$-values of the Cox models. For this reason, the mean values between cases and non-cases were used to interpret our results in Table 1 and the $P$-values were not displayed. Finally, we were not able to adjust for contaminants such as $\mathrm{Hg}$ (found in fish) and persistent organic pollutants (POP, the major sources of which are fish, meat and dairy products) that have been associated with incident T2D in some studies $^{(45-47)}$. However, epidemiological evidence on POP has been considered inconsistent enough that studies suggest that the potential hazards of the low levels of exposure present in seafood do not outweigh the benefits of seafood consumption. In addition, meta-analyses in East Asian countries where fish consumption is high even demonstrate a decreased risk for T2D associated with a high fish consumption ${ }^{(48,49)}$. Yet, interpretation of the findings should be cautious in the absence of adjustment for contaminants. The strengths of this study include the prospective design that minimises recall and selection biases and the large study population. The high rate of follow-up allowed the validation of many cases of incident T2D, and the dietary questionnaire was validated and specific for this French population. Extensive information on potential confounders was collected, minimising residual confounding. We also controlled for other FA groups besides the one in question in our analyses. This is important, as the fate of FA depends heavily on the overall FA profile of the diet, likely due to competition between FA for enzymes ${ }^{(14)}$.

\section{Conclusion}

Despite the large body of evidence suggesting that PUFA, and specifically n-3 PUFA, are beneficial for cardiovascular health ${ }^{(50)}$, based on our results we could not recommend a high consumption of either as beneficial for diabetes prevention, as the effects of PUFA appear heterogeneous within the group and even within subgroups. High AA and DPA intakes were associated with an increased risk for incident T2D, independently of meat consumption, the primary food group contributor. The association could be even more pronounced in the general population, as our population is relatively homogeneous. Further investigation into the associations of individual dietary $n-3$ and $n-6$ PUFA intake and the risk for T2D is warranted.

\section{Acknowledgements}

The authors are grateful to the participants of $\mathrm{E} 3 \mathrm{~N}$ for their continued participation and dedication in the $\mathrm{E} 3 \mathrm{~N}$ cohort.

This study was supported by the Cardiovasculaire, Obésité, Rein, Diabète (CORDDIM) Program. CORDDIM had no role in the design, analysis or writing of this article.
G. F., M. M. and C. D. designed the research; M. M. and C. D. analysed the data or performed the statistical analysis; C. D. wrote the paper; G. F. had primary responsibility for the final content. F. C.-C., F. B., B. B., A. A. and M.-C. B.-R. reviewed and commented on the final manuscript. All the authors read and approved the final manuscript.

The authors declare that there are no conflicts of interest.

\section{Supplementary materials}

For supplementary material/s referred to in this article, please visit https://doi.org/10.1017/S0007114516003883

\section{References}

1. Hu FB, Manson J, Stampfer M, et al. (2001) Diet, lifestyle, and the risk of type 2 diabetes mellitus in women. $N$ Engl J Med 345, 790-797.

2. Steinbrecher A, Morimoto Y, Heak S, et al. (2011) The preventable proportion of type 2 diabetes by ethnicity: the multiethnic cohort. Ann Epidemiol 21, 526-535.

3. McKinley Health Center (2015) Macronutrients: the importance of carbohydrate, protein, and fat. http://www.mckinley.illinois. edu/handouts/macronutrients.htm (accessed March 2016).

4. Hu J, La Vecchia C, de Groh M, et al. (2011) Dietary transfatty acids and cancer risk. Eur J Cancer Prev 20, 530-538.

5. Mozaffarian D, Aro A \& Willett WC (2009) Health effects of trans-fatty acids: experimental and observational evidence. Eur J Clin Nutr 63, Suppl. 2, S5-S21.

6. Schwab U, Lauritzen L, Tholstrup T, et al. (2014) Effect of the amount and type of dietary fat on cardiometabolic risk factors and risk of developing type 2 diabetes, cardiovascular diseases, and cancer: a systematic review. Food Nutr Res 58, 25145.

7. Salmerón J, Hu FB, Manson JE, et al. (2001) Dietary fat intake and risk of type 2 diabetes in women. Am J Clin Nutr $\mathbf{7 3}$, 1019-1026.

8. Meyer KA, Kushi LH, Jacobs D Jr, et al. (2001) Dietary fat and incidence of type 2 diabetes in older Iowa women. Diabetes Care 24, 1528-1535.

9. Virtanen JK, Mursu J, Voutilainen S, et al. (2014) Serum omega-3 polyunsaturated fatty acids and risk of incident type 2 diabetes in men: the Kuopio Ischemic Heart Disease Risk Factor study. Diabetes Care 37, 189-196.

10. Forouhi NG, Koulman A, Sharp SJ, et al. (2014) Differences in the prospective association between individual plasma phospholipid saturated fatty acids and incident type 2 diabetes: the EPIC-InterAct case-cohort study. Lancet Diabetes Endocrinol 2 , 810-818.

11. Zheng JS, Huang T, Yang J, et al. (2012) Marine $n-3$ polyunsaturated fatty acids are inversely associated with risk of type 2 diabetes in Asians: a systematic review and metaanalysis. PLOS ONE 7, e44525.

12. Wallin A, Di GD, Orsini N, et al. (2012) Fish consumption, dietary long-chain $n-3$ fatty acids, and risk of type 2 diabetes: systematic review and meta-analysis of prospective studies. Diabetes Care 35, 918-929.

13. Wu JH, Micha R, Imamura F, et al. (2012) Omega-3 fatty acids and incident type 2 diabetes: a systematic review and metaanalysis. Br J Nutr 107, Suppl. 2, S214-S227.

14. Siguel EN \& Maclure M (1987) Relative activity of unsaturated fatty acid metabolic pathways in humans. Metabolism $\mathbf{3 6}$, 664-669. 
15. van Liere MJ, Lucas F, Clavel F, et al. (1997) Relative validity and reproducibility of a French dietary history questionnaire. Int J Epidemiol 26, Suppl. 1, S128-S136.

16. Willett W \& Stampfer MJ (1986) Total energy intake: implications for epidemiologic analyses. Am J Epidemiol 124, 17-27.

17. Lajous M, Tondeur L, Fagherazzi G, et al. (2012) Processed and unprocessed red meat consumption and incident type 2 diabetes among French women. Diabetes Care 35, 128-130.

18. Cottet V, Touvier M, Fournier A, et al. (2009) Postmenopausal breast cancer risk and dietary patterns in the E3N-EPIC prospective cohort study. Am J Epidemiol 170, 1257-1267.

19. van Dam RM, Willett WC, Rimm EB, et al. (2002) Dietary fat and meat intake in relation to risk of type 2 diabetes in men. Diabetes Care 25, 417-424.

20. Micha R \& Mozaffarian D (2010) Saturated fat and cardiometabolic risk factors, coronary heart disease, stroke, and diabetes: a fresh look at the evidence. Lipids 45, 893-905.

21. Lankinen MA, Stancáková A, Uusitupa M, et al. (2015) Plasma fatty acids as predictors of glycaemia and type 2 diabetes. Diabetologia 58, 2533-2544.

22. Lemaitre RN, Fretts AM, Sitlani CM, et al. (2015) Plasma phospholipid very-long-chain saturated fatty acids and incident diabetes in older adults: the Cardiovascular Health Study. Am J Clin Nutr 101, 1047-1054.

23. Ameer F, Scandiuzzi L, Hasnain S, et al. (2014) De novo lipogenesis in health and disease. Metabolism 63, 895-902.

24. United States Department of Agriculture (2016) National nutrient database for standard reference release 28. http:// ndb.nal.usda.gov/ndb/foods/show $/ 660 ?$ fgcd $=\&$ man $=\& l f a c e t=$ $\&$ count $=\& \max =35 \&$ sort $=\&$ qlookup $=$ olive + oil $\&$ offset $=\&$ format $=$ Full\&new $=\&$ measureby $=($ accessed February 2016)

25. Violi F, Loffredo L, Pignatelli P, et al. (2015) Extra virgin olive oil use is associated with improved post-prandial blood glucose and LDL cholesterol in healthy subjects. Nutr Diabetes 5, e172.

26. Guasch-Ferre M, Hruby A, Salas-Salvado J, et al. (2015) Olive oil consumption and risk of type 2 diabetes in US women. Am J Clin Nutr 102, 479-486.

27. Morio B, Fardet A, Legrand P, et al. (2016) Involvement of dietary saturated fats, from all sources or of dairy origin only, in insulin resistance and type 2 diabetes. Nutr Rev 74, 33-47.

28. Gijsbers L, Ding EL, Malik VS, et al. (2016) Consumption of dairy foods and diabetes incidence: a dose-response meta-analysis of observational studies. Am J Clin Nutr 103, 1111-1124.

29. Hodge AM, English DR, O'Dea K, et al. (2007) Plasma phospholipid and dietary fatty acids as predictors of type 2 diabetes: interpreting the role of linoleic acid. Am J Clin Nutr $\mathbf{8 6}$, 189-197.

30. Meyer BJ, Mann NJ, Lewis JL, et al. (2003) Dietary intakes and food sources of omega-6 and omega-3 polyunsaturated fatty acids. Lipids 38, 391-398.

31. Wang Q, Imamura F, Ma W, et al. (2015) Circulating and dietary trans fatty acids and incident type 2 diabetes in older adults: the Cardiovascular Health Study. Diabetes Care 38, 1099-1107.

32. Mozaffarian D, Cao H, King IB, et al. (2010) Trans-palmitoleic acid, metabolic risk factors, and new-onset diabetes in U.S. adults: a cohort study. Ann Intern Med 153, 790-799.
33. Alberts B, Bray D, Lewis J, et al. (2002) Membrane structure. In Molecular Biology of the Cell. New York: Garland Science.

34. Andersson A, Nalsen C, Tengblad S, et al. (2002) Fatty acid composition of skeletal muscle reflects dietary fat composition in humans. Am J Clin Nutr 76, 1222-1229.

35. Raatz SK, Bibus D, Thomas W, et al. (2001) Total fat intake modifies plasma fatty acid composition in humans. J Nutr $\mathbf{1 3 1}$, 231-234.

36. Weijers RN (2012) Lipid composition of cell membranes and its relevance in type 2 diabetes mellitus. Curr Diabetes Rev $\mathbf{8}$, 390-400.

37. Borkman M, Storlien L, Pan D, et al. (1993) The relation between insulin sensitivity and the fatty acid composition of skeletal muscle phospholipids. $N$ Engl J Med 328, 238-244.

38. Ansorena D, Guembe A, Mendizabal T, et al. (2010) Effect of fish and oil nature on frying process and nutritional product quality. J Food Sci 75, H62-H67.

39. Clarke SD, Baillie R, Jump DB, et al. (1997) Fatty acid regulation of gene expression. Its role in fuel partitioning and insulin resistance. Ann N Y Acad Sci 827, 178-187.

40. Itoh Y, Kawamata Y, Harada M, et al. (2003) Free fatty acids regulate insulin secretion from pancreatic beta cells through GPR40. Nature 422, 173-176.

41. Band AM, Jones PM \& Howell SL (1993) The mechanism of arachidonic acid-induced insulin secretion from rat islets of Langerhans. Biochim Biophys Acta 1176, 64-68.

42. Galgani JE, Aguirre CA, Uauy RD, et al. (2007) Plasma arachidonic acid influences insulin-stimulated glucose uptake in healthy adult women. Ann Nutr Metab 51, 482-489.

43. Brenner RR (2003) Hormonal modulation of delta 6 and delta5 desaturases: case of diabetes. Prostaglandins Leukot Essent Fatty Acids 68, 151-162.

44. Kaur G, Cameron-Smith D, Garg M, et al. (2011) Docosapentaenoic acid (22:5n-3): a review of its biological effects. Prog Lipid Res 50, 28-34.

45. World Health Organization (2014) Dioxins and their effects on human health. http://www.who.int/mediacentre/factsheets/ fs225/en/ (accessed July 2016).

46. Wu H, Bertrand KA, Choi AL, et al. (2013) Persistent organic pollutants and type 2 diabetes: a prospective analysis in the nurses' health study and meta-analysis. Environ Health Perspect 121, 153-161.

47. He K, Xun P, Liu K, et al. (2013) Mercury exposure in young adulthood and incidence of diabetes later in life: the CARDIA Trace Element Study. Diabetes Care 36, 1584-1589.

48. Lee DH \& Jacobs DR Jr (2010) Inconsistent epidemiological findings on fish consumption may be indirect evidence of harmful contaminants in fish. J Epidemiol Community Health 64, 190-192.

49. Xun P \& He K (2012) Fish consumption and incidence of diabetes: meta-analysis of data from 438,000 individuals in 12 independent prospective cohorts with an average 11-year follow-up. Diabetes Care 35, 930-938.

50. Mozaffarian D \& Wu JH (2011) Omega-3 fatty acids and cardiovascular disease: effects on risk factors, molecular pathways, and clinical events. J Am Coll Cardiol 58, 2047-2067. 